\title{
Atrioventricular septal defect- the need for a flexible surgical approach in a lesion with markedly individual features
}

\author{
Yves Lecompte and Giancarlo Crupi
}

$\mathbf{H}$ earts with biventricular connections and deficient atrioventricular septation are readily recognizable from their characteristic morphology. ${ }^{1}$ As yet, however, there is still no consensus on precisely what to call them, ${ }^{2}$ how to categorize them, and, perhaps most importantly, how to approach their surgical repair.

Following important articles concerned with diagnosis and assessment in both fetal and neonatal life, a large part of this issue of Cardiology in the Young is concerned with surgical management of this lesion. This provides ample confirmation of what any surgeon has learnt from his own experience-atrioventricular septal defect remains a difficult lesion to repair. This is not because the many morphologic variants of this lesion are not appreciated to their full extent. Rather it can be said, quite simply, that no one case is ever identical to another. As a consequence, a uniform satisfactory repair of this anomaly is difficult to achieve. Not infrequently, further and more complex reoperations are required both early and late following the initial operation.

It should also come as no surprise to notice that the concepts expressed in this brief introduction are not accompanied by hard, statistically significant, data. Indeed, the comments are "impressions" born from the everyday experience of two surgeons working in different places, who have in common the same perception of the problems and of the difficulties encountered in the surgical management of this anomaly. We only wish to share our thoughts with the readers of this journal, aiming at stimulating their ingenuity in the search for a type of repair which will prove most satisfactory in their own practice.

In this respect, we support most strongly the concept expressed by Ebels, ${ }^{3}$ namely that the surgical approach for repair of atrioventricular septal defect should be "individualized", being based on the specific anatomy encountered in each case. At the moment, however, the flexibility offered for the management of the patients with this lesion seems to be limited mostly to the choice of whether to use a single patch as opposed to a double patch to repair the septal defect, and whether or not to close the septal commissure of the left atrioventricular valve ("mitral" cleft). Do such limited choices still make sense? Probably not, and for at least two reasons. Firstly, each type of repair can be employed with equally satisfactory results, as shown by the various surgical reports described in this issue. ${ }^{4-7}$ Secondly, it is always difficult to predict the adequacy of a specific type of repair from preoperative evaluation. This is particularly true with regard to the left atrioventricular valve.

To achieve an optimal repair, it is crucial to understand the changes which occur in the function of the left atrioventricular valve following closure of the ventricular and atrial components of the septal defect and resuspension of the leaflets of the valve itself. This maneuver will inevitably restrict the motion of the bridging leaflets regardless of the surgical technique employed.

Along with Ebels, ${ }^{3}$ we believe that it is the function of the left atrioventricular valve which should dictate the type of repair attempted rather than any preconceived idea of what this valve should look like, be that notion based on either a bileaflet or a trifoliate model. It is, therefore, very important to provide further studies on the postoperative pathophysiology, studies which would be of more practical value than those concerned with preoperative function, this feature inevitably to be modified by the surgical repair.

In the absence of such studies, the surgeon must choose the type of repair to be employed without having a well-defined criterion for making the decision. It is our belief that this choice should be made on an individual basis, but it still remains difficult to achieve the mental flexibility which is required to determine the most appropriate type of repair for each individual patient. Despite this, it can be stated with certainty that repair of the left atrioventricular valve and avoidance of obstruction to the subaortic outflow tract remain two of 
the most controversial problems in the surgical management of atrioventricular septal defect.

\section{Repair of the left atrioventricular valve}

It is perhaps not generally appreciated that, prior to surgery, and particularly when repair is performed in the first six months of life, the function of the left atrioventricular valve in most patients is normal. ${ }^{5,6}$ In these instances, therefore, the left atrioventricular valve should not require any surgical handling at all apart from resuspension from the patch used for closure of the ventricular component of the septal defect. Yet as already pointed out, it is always difficult to anticipate to what extent this maneuver interferes with the valve motion which, inevitably, will be different from that observed prior to repair. Indeed, despite all efforts, identification of the optimal level of resuspension of the superior and inferior bridging leaflers of the left atrioventricular valve is rather arbitrary and likely to be less than perfect. This may explain why, after surgical repair, regurgitation may occur in patients in whom the left atrioventricular valve was entirely competent prior to surgery.

The function of the left atrioventricular valve, therefore, should be carefully evaluated not only before, but especially after, resuspension. One of the most frequent difficulties encountered in achieving competence of the left atrioventricular valve is the lack of valvar tissue at the level of one of the bridging leaflets, generally the inferior one. This problem may be further complicated by the presence of short tendinous cords tethering the free edge of the leaflet to the crest of ventricular septum and, therefore, restricting the motion of the leaflets and preventing satisfactory coaptation with the mural leaflet. We believe that division of these cords, even if they are deemed to be primary cords, is the only way of restoring normal motion of the valve and augmenting its surface. Following such a maneuver, however, suture of the septal commissure is mandatory so as to prevent massive postoperative regurgitation due to mobilization of the bridging leaflets.

In contrast, when the coaptation of the bridging leaflets with each other and with the mural leaflet is satisfactory after resuspension, it may be justifiable to leave open the septal commissure. This will avoid any excessive reduction of valvar mobility which, if produced, may eventually result in stenosis. The decision of whether or not to close the septal commissure, therefore, should be made only after careful evaluation of the need for mobilization of the bridging leaflets by division of their cordal attachments to the crest of the ventricular septum. It is somehow unfortunate that this decision has to be made before closure of the ventricular septal defect. The relative size of the mural leaflet, a feature emphasized by Ebels ${ }^{3}$ and Abruzzese, ${ }^{7}$ is probably an important criterion when deciding the fate of the septal commissure. This, nonetheless, is only one of the elements to be studied. The ideal technique should probably combine some maneuvers aimed at increasing the valvar motion with others aimed at restricting it. In this regard, the "liberal" use of annuloplasties as suggested by Laks ${ }^{5}$ may be extremely useful in achieving maximal competence. It must also be stated that the "case by case" strategy is by no means original. This is what we have learnt from Carpentier and other pioneers in the general field of reconstructive valvar surgery. There is no reason why it should not equally be applied to the surgical management of atrioventricular septal defects.

\section{The left ventricular outflow tract}

The risk of creating subaortic stenosis after repair of atrioventricular septal defect is, nowadays, well recognized. Little attention, however, has been paid by surgeons to this problem because, as stated by Ebels, ${ }^{3}$ the left ventricular outflow tract is generally not seen during repair. Overt obstruction is also remarkably rare regardless of the preoperative extent of narrowing of the left ventricular outflow tract, and of the type of repair performed, including suture of the septal commissure. As Anderson and colleagues indicate, ${ }^{1}$ this complication is much more likely to occur in hearts with separate atrioventricular orifices. This is of extreme importance, since the lesion with separate valvar orifices is generally treated by suture of the septal commissure and closure of the atrial component of the septal defect. This absence of concern is probably the consequence of the erroneous separation which, following tradition, still compartments the forms of atrioventricular septal defect with a common atrioventricular orifice (considered to be a serious disease) from those with two atrioventricular orifices, the latter being considered more benign. We must now question this conventional wisdom.

It would, of course, be of most value were the surgeon to have precise preoperative criteria with which to predict the risk of creating postoperative subaortic obstruction. Unfortunately, these criteria do not exist. We know, however, that the risk is dependent mostly upon the mode of attachment of the superior bridging leaflet to the crest of the interventricular septum. We believe ourselves that the risk of producing obstruction is more important when the tethering of the superior bridging leaflet to the crest of the ventricular septal defect is produced by many short tendinous cords 
which obliterate completely the ventricular septal defect. In thirteen such cases encountered in our services, cases which were referred with the diagnosis of "partial atrioventricular canal", we detached, as previously suggested by Chang and Becker, ${ }^{8}$ the superior bridging leaflet from the crest of the atrioventricular septal defect. In this way, a so-called complete defect was created, permitting placement of an interventricular patch for enlargement of the subaortic outflow tract. Repair was uneventful in each case. We believe that the same technique can be employed when the ventricular septal defect is not completely obliterated, leaving several small interventricular communications between the tendinous cords (so-called intermediate form of atrioventricular canal). Yet, it must be recognized that the precise indications for this type of repair are still uncertain.

\section{Conclusion}

Surgical repair of atrioventricular septal defects has, in the past, perhaps been oversimplified because of the complexity of the lesion and because of the attempt to find precise relationships between precise anatomic and physiologic categories and techniques of repair where none exist. Even if less than perfect, nonetheless, we must recognize that this approach has been associated with increasingly satisfactory results. Despire this, we all feel that much progress remains to be made. The contents of this issue of Cardiology in the Young may well point towards the path to follow. We must abandon the security of the old anatomic classifications which provided limited categories into which, like Procrustes, we tried to compress all our cases. We must also dissociate the physiologic status from these preconceived anatomic categories. Most importantly, we must free ourselves from the dogmas of standardized repair following the tenets of a particular surgical school. Instead, we must assess each case on its own merits, judging the most appropriate surgical repair on the results of both preoperative and postoperative evaluation. Hopefully, such an individualized approach will result in a new form of simplicity rather than an increased complexity which might be anticipated.

Centre Cardio-Thoracic de Monaco

11 bis Avenue d'Ostende

B.P. 223

MO 98004, Monaco Cedéx

Monte Carlo

Department of Cardiac Surgery

Ospedali Riuniti di Bergamo

Largo Barozzi 1

24100 Bergamo

Italy

\section{References}

1. Anderson RH, Baker EJ, Ho SY, Rigby ML, Ebels T. The morphology and diagnosis of atrioventricular septal defects. Cardiol Young 1991; 1: 290-305.

2. Becker AE, Anderson RH. Atrioventricular septal defects. What's in a name? J Thorac Cardiovasc Surg 1982; 83: 484489.

3. Ebels T. Surgery of the atrioventricular valve and of the left ventricular outflow tract in atrioventricular septal defect. Cardiol Young 1991; 1: 344-355.

4. Merrill WH, Hammon JW Jr, Bender HW Jr. Technique of repair of complete atrioventricular septal defect. Cardiol Young 1991; $1: 379-382$.

5. Laks H, Capouya ER, Pearl JM, Elami A, Drinkwater DC. Technique of management of the left atrioventricular valve in the repair of atrioventricular septal defect with a common atrioventricular orifice. Cardiol Young 1991; 1: 356-366.

6. Lacour-Gayet F, Comas J, Bruniaux J, Serraf A, Losay J, Petit J, Dervanian P, Planché C. Management of the left atrioventricular valve in 95 parients with atrioventricular septal defects and a common atrioventricular orifice. A ten year review. Cardiol Young 1991; 1: 367-373.

7. Abbruzzese PA, Crupi G, Tumbarello R, Napoleone A, Merlo $M$, Parenzan L. Atrioventricular septal defect. The left atrioventricular valve at reoperation. Cardiol Young 1991; 1:374378.

8. Chang CI, Becker AE. Surgical anatomy of left ventricular outflow tract obstruction in complete atrioventricular septal defect. A concept for operative repair. J Thorac Cardiovasc Surg 1987; 94: 897-903. 